\title{
Effect of Neoadjuvant Chemotherapy on Renal Function following Radical Cystectomy: Is there a Meaningful Impact?
}

\author{
Thenappan Chandrasekar ${ }^{\mathrm{a}, *}$, Neil Pugashetti ${ }^{\mathrm{b}}$, Blythe Durbin-Johnson ${ }^{\mathrm{c}}$, Marc A. Dall'Era ${ }^{\mathrm{b}, \mathrm{d}}$, \\ Christopher P. Evans ${ }^{\mathrm{b}, \mathrm{d}}$, Ralph W. deVere White ${ }^{\mathrm{b}, \mathrm{d}}$ and Stanley A. Yap ${ }^{\mathrm{b}, \mathrm{d}}$ \\ ${ }^{a}$ Departments of Surgery and Surgical Oncology, Division of Urology, Princess Margaret \\ Cancer Centre, University Health Network and the University of Toronto, Toronto, ON, Canada \\ ${ }^{\mathrm{b}}$ Department of Urology, University of California, Davis, Sacramento, CA, USA \\ ${ }^{\mathrm{c}}$ Department of Public Health Sciences, Division of Biostatistics, University of California \\ Davis, Davis, CA, USA \\ ${ }^{\mathrm{d}}$ University of California Davis Comprehensive Cancer Center, Sacramento, CA, USA
}

\begin{abstract}
.
Objective: To evaluate the patterns of impact of neoadjuvant chemotherapy (NAC) on renal function across the initial year following treatment for muscle-invasive bladder cancer (MIBC) with radical cystectomy (RC).

Methods: We reviewed the charts of 241 patients who underwent RC for urothelial carcinoma of the bladder between $2003-14$ at our institution. Renal function was evaluated at multiple time points (pre-chemotherapy, pre-operatively, post-operatively, 6-12 months follow-up), and then classified by CKD staging. Univariable and multivariable logistic regression analyses were performed to determine relationship between NAC and change in CKD stage.

Results: Of the 241 patients who underwent RC for urothelial carcinoma of the bladder, 66 (27\%) received NAC and $175(73 \%)$ did not. In multivariable analysis, NAC was significantly associated with a decrease of at least one CKD stage from baseline to post-op ( $p=0.009$ ), but not to the 6-12 months follow-up time point $(p=0.050)$. The loss of GFR in the NAC cohort occurs up-front with chemotherapy, but the peri-operative course is similar to those who underwent cystectomy alone. Of the 15 NAC patients $(26.8 \%)$ who were Stage 3 CKD prior to chemotherapy, none progressed to a higher stage CKD.

Conclusion: NAC is associated with an initial decline in GFR, which then remains stable through the first year following RC. Despite an initial insult, patients receiving NAC are not vulnerable to further deterioration. When appropriately selected, NAC does not appear to result in a clinically significant deterioration of renal function.
\end{abstract}

Keywords: Urinary bladder neoplasms, renal insufficiency, acute kidney injury, neoadjuvant therapy, antineoplastic agents

\footnotetext{
${ }^{*}$ Correspondence to: Thenappan Chandrasekar, Division of Urologic Oncology, Department of Surgical Oncology, University Heath Network, OPG Building (Queen's Park Subway Station),
}

700 University Avenue, 6th Floor, Toronto, ON, M5G 1Z5, CA. Tel.: +1 732742 1025; Fax: +1 844351 9508; E-mail: thenappan.chandrasekar@gmail.com. 


\section{INTRODUCTION}

Urothelial carcinoma of the bladder is the fourth most common malignancy in the United States, and mortality in this disease is primarily driven by those with muscle-invasive bladder cancer (MIBC) [1]. Overall 5-year survival in patients with MIBC is approximately $50 \%$, and this has remained relatively unchanged for the past 20 years [2]. A major shift in the management of MIBC has been the introduction of platinum-based neoadjuvant chemotherapy prior to radical cystectomy for clinically localized disease (cT2-T4aNOM0), which has now been incorporated into international guidelines as the standard of care for eligible patients.

The standard chemotherapy regimens utilized for neoadjuvant chemotherapy have traditionally been MVAC (methotrexate, vinblastine, doxorubicin, and cisplatin) or gemcitabine/cisplatin [3]. Recently, there has also been the introduction of a shorter course of MVAC, entitled dose-dense or accelerated MVAC (ddMVAC or AMVAC) [4]. While cisplatin is the cornerstone of chemotherapy for bladder cancer, it is often substituted with the less nephrotoxic carboplatin in patients with renal insufficiency; however, oncologic outcomes have not been demonstrated to be equivalent to cisplatin $[5,6]$.

Utilization of neoadjuvant chemotherapy for MIBC, despite organizational and guideline recommendations for its use, remains low. Zaid $\mathrm{HB}$ et al. demonstrated that NAC utilization in 2010 among eligible patients in the United States was around 21\%; by 2015 , this number had improved to approximately $40 \%$ in a Canadian cohort $[7,8]$. These studies indicate that the majority of eligible patients are not receiving therapy that could potentially provide survival benefit. While the reasons for underutilization are likely multifactorial, concern about the effects of chemotherapy prior to surgery may contribute to the decision to not pursue NAC $[9,10]$, particularly due to the known nephrotoxicity of cisplatin-based chemotherapy [9]. Furthermore, radical cystectomy itself has been associated with long-term deterioration of renal function; as such, the concern for two nephrotoxic events may contribute to the hesitation to utilize NAC [11-14]. Despite these concerns, there have been no studies evaluating the impact of neoadjuvant chemotherapy on renal function following radical cystectomy. Utilizing our institutional database, we aim to address this deficit in the literature by following renal function metrics in patients treated with radical cystectomy and neoadjuvant chemotherapy.

\section{METHODS}

\section{Patient selection and data collection}

We utilized an institutional database encompassing all patients treated with open radical cystectomy at the University of California, Davis Medical Center between 2003-2014. Comprehensive chart review was performed including review of demographic information, medical and surgical history, clinic and hospital admission notes, pathology reports, and laboratory reports. Patients were identified as to whether or not they received NAC. Chemotherapy regimen and duration was assessed. Individual patient comorbidities were identified, including diabetes mellitus, hypertension, obesity (body mass index), prior cerebrovascular accident (stroke), prior myocardial infarction, prior venous thromboembolism (DVT or PE), and smoking status.

Data was only abstracted from patients with a diagnosis of urothelial carcinoma (UC) on the final pathologic specimen from cystectomy (or pT0 cystectomy pathology and UC on initial biopsy); patients with histologic types other than UC were excluded (36 patients). Only patients with a diagnosis of muscle-invasive UC who received preoperative systemic chemotherapy were included in the NAC and RC cohort.

Renal function (serum creatinine, glomerular filtration rate using the Modification of Diet in Renal Disease (MDRD) equation) was documented at multiple time points: pre-chemotherapy (if applicable), pre-operatively, post-operatively, and at 6-12 months follow-up. These were then classified by chronic kidney disease (CKD) staging according to the National Kidney Foundation's Kidney Disease Outcomes Quality Initiative [15].

\section{Statistical analysis}

Overall cohort characteristics were determined and reported based on whether or not NAC was utilized. Groups were compared across baseline variables using chi-square tests for categorical baseline variables and $t$-tests for continuous baseline variables (with follow-up time log transformed before testing). Univariable analyses were conducted examining the effect of each patient or treatment characteristic on renal function outcomes. Patient or treatment characteristics with a $p$-value less than 0.20 in univariable analysis were included in a multivariable model of each renal function outcome, with neoadjuvant 
chemotherapy identified as the primary effector variable. Diabetes mellitus and hypertension, established risk factors for renal deterioration, were also included in the multivariable models, regardless of univariable analysis. Categorical outcomes related to renal function (worsening from CKD 1-2 to 3-5, and worsening by one grade or more) were analyzed using logistic regression. Analyses of worsening from CKD 1 or 2 to 3 or higher only included patients with grades 1 or 2 at baseline.

Initial analysis was focused on the entire cohort, utilizing the worsening of CKD stage by one stage or more as the categorical outcome. A subset analysis was completed on the patients with abnormal baseline renal function (CKD stage 3-5) prior to initiating chemotherapy.

This study was approved by the Institutional Review Board at the University of California, Davis. All analyses were conducted using $\mathrm{R}$, version 3.1.1 (R Core Team, 2014).

\section{RESULTS}

Of the 241 patients with urothelial carcinoma of the bladder who underwent radical cystectomy (RC) at our institution, 66 (27\%) received neoadjuvant chemotherapy (NAC) prior to RC and 175 (73\%) underwent RC alone. Table 1 reviews the demographics of the two cohorts. Patients who underwent neoadjuvant chemotherapy and radical cystectomy tended to be younger (65.9 years old vs. 68.4 years old, $p=0.05$ ) and have slightly less follow-up (116.8 weeks vs. 120.1 week, $p=0.05$ ), but had a much higher rate of complete response (pT0) after cystectomy $(21.2 \%$ vs. $9.1 \%, p=0.012)$. There were no significant differences in comorbidities such as smoking status, diabetes mellitus, coronary artery disease, prior myocardial infarction (MI), prior cardiovascular accident (CVA), or prior venous thromboembolism (VTE). Diversion type was not associated with having received neoadjuvant chemotherapy.

Of the 66 patients, $42(63.6 \%)$ received cisplatin based chemotherapy and $15(22.7 \%)$ received carboplatin based chemotherapy. Nine (13.6\%) received alternative or unknown regimens $-1(1.5 \%)$ was switched from cisplatin to carboplatin during treatment, $2(3.0 \%)$ received alternative regimens, and $6(9.1 \%)$ received an unknown regimen. Of the 42 who received cisplatin-based chemotherapy, 6 received MVAC (methotrexate, vinblastine, doxorubicin, cisplatin) and 35 received GC (gemcitabine, cisplatin).

Table 2 details the renal function outcomes (mean serum creatinine, mean GFR, and CKD stage classification) of the patients at each of the major time points.

Our initial analysis focused on change in CKD stage, with a change being defined as an increase of at least one CKD stage. Univariate logistic regression analysis was then performed assessing change in CKD stage from baseline (pre-chemotherapy in NAC/RC cohort, pre-operative in $\mathrm{RC}$ only cohort) to post-operative and 6-12 follow-up time points (Supplementary Table 1). In the multivariable logistic regression analysis, accounting for all factors identified in univariate analysis, neoadjuvant chemotherapy was an independent predictor of worsening of at least one CKD stage from baseline to the post-operative time point (OR 2.486, $p=0.009)$, but not to the 6-12 month follow-up time point (OR 2.497, $p=0.05$ ) (Table 3A). However, when looking specifically at patients who received cisplatin-based NAC compared to carboplatin-based NAC, cisplatin-based NAC was an independent predictor of worsening of at least one CKD stage from baseline to the post-operative time point (OR 3.726, $p=0.001)$ and to the 6-12 month follow-up time point (OR 3.313, $p=0.017$ ), while carboplatin-based NAC was not (OR 0.301, $p=0.261 ; 0.223, p=0.219$, respectively) (Table 3B).

We then specifically addressed the subset of patients with normal renal function (CKD 1-2) prior to any neoadjuvant therapy or surgery. Initially, we assessed for any worsening of CKD stage. Univariate logistic regression analysis identified NAC as a significant predictor of worsening CKD stage from baseline to post-operatively (OR $2.175, p=0.046$ ), which was confirmed on multivariable logistic regression (OR 2.226, $p=0.044$ ). However, neoadjuvant chemotherapy was not a significant predictor of worsening CKD stage from baseline to 6-12 months follow-up on univariate or multivariable analysis (OR 1.836, $p=0.278$ ) (Supplementary Table 2).

In an effort to address the most clinically relevant question, we then focused on identifying clinically significant renal function loss. CKD stage was categorized into a dichotomous variable: normal (CKD 1-2) or abnormal (CKD 3-5). Identifying the cohort of patients with normal renal function at baseline (CKD 1-2), we identified predictors of progression to abnormal renal function (CKD 3-5). NAC was associated with a trend towards 
Table 1

Patient demographics

\begin{tabular}{|c|c|c|c|}
\hline & $\begin{array}{l}\text { RC Alone } \\
(n=175)\end{array}$ & $\begin{array}{l}\text { NAC plus RC } \\
\quad(n=66)\end{array}$ & $P$-Value \\
\hline \multicolumn{4}{|l|}{ Age at Surgery (Years) } \\
\hline Mean (SD) & $68.4(9.3)$ & $65.9(8.7)$ & \multirow[t]{2}{*}{0.05} \\
\hline Median (IQR) & $70(62-76)$ & $66(61-72)$ & \\
\hline \multicolumn{4}{|c|}{ Follow-up from Date of diagnosis (Months) } \\
\hline Mean (SD) & $30(30)$ & $29.2(26.3)$ & \multirow[t]{2}{*}{0.05} \\
\hline Median (IQR) & $17.4(5.4-45.8)$ & $19.7(11.8-38.3)$ & \\
\hline \multicolumn{4}{|l|}{$\operatorname{Sex}(N, \%)$} \\
\hline Female & $36(20.6 \%)$ & $12(18.2 \%)$ & \multirow[t]{2}{*}{0.82} \\
\hline Male & $139(79.4 \%)$ & $54(81.8 \%)$ & \\
\hline \multicolumn{4}{|l|}{ Smoking Status $(N, \%)$} \\
\hline Non-Smoker & $30(17.1 \%)$ & $12(18.2 \%)$ & \multirow[t]{3}{*}{0.47} \\
\hline Former Smoker & $111(63.4 \%)$ & $36(54.5 \%)$ & \\
\hline Active Smoker & $32(18.3 \%)$ & $16(24.2 \%)$ & \\
\hline \multicolumn{4}{|l|}{ Pre-op Diabetes Mellitus (N, \%) } \\
\hline No & $144(82.3 \%)$ & $56(84.8 \%)$ & \multirow[t]{2}{*}{0.55} \\
\hline Yes & $29(16.6 \%)$ & $8(12.1 \%)$ & \\
\hline \multicolumn{4}{|c|}{ Pre-op Coronary Artery Disease (N, \%) } \\
\hline No & $145(82.9 \%)$ & $53(80.3 \%)$ & \multirow[t]{2}{*}{1.00} \\
\hline Yes & $28(16 \%)$ & $11(16.7 \%)$ & \\
\hline \multicolumn{4}{|c|}{ Pre-op Myocardial Infarction (N, \%) } \\
\hline No & $157(89.7 \%)$ & $60(90.9 \%)$ & \multirow[t]{2}{*}{0.64} \\
\hline Yes & $16(9.1 \%)$ & $4(6.1 \%)$ & \\
\hline \multicolumn{4}{|c|}{ Pre-op Cerebrovascular Accident $(N, \%)$} \\
\hline No & $165(94.3 \%)$ & $62(93.9 \%)$ & \multirow[t]{2}{*}{0.88} \\
\hline Yes & $8(4.6 \%)$ & $2(3 \%)$ & \\
\hline \multicolumn{4}{|c|}{ Pre-op Venous Thromboembolism (N, \%) } \\
\hline No & $163(93.1 \%)$ & $58(87.9 \%)$ & \multirow[t]{2}{*}{0.49} \\
\hline Yes & $10(5.7 \%)$ & $6(9.1 \%)$ & \\
\hline \multicolumn{4}{|c|}{ Preoperative Clinical T-Stage $(c T)(N, \%)$} \\
\hline $\mathrm{cTa}$, cTis, or cT1 & $64(36.6 \%)$ & 0 & \multirow[t]{2}{*}{$<0.001$} \\
\hline $\mathrm{cT} 2-\mathrm{cT} 4$ & $110(62.9 \%)$ & $66(100 \%)$ & \\
\hline \multicolumn{4}{|c|}{ Postoperative Pathologic T-Stage $(p T)(N, \%)$} \\
\hline pT0 & $16(9.1 \%)$ & $12(21.2 \%)$ & \multirow[t]{4}{*}{0.01} \\
\hline pTa, pTis, or pT1 & $52(29.7 \%)$ & $9(13.6 \%)$ & \\
\hline pT2 & $21(12 \%)$ & $10(15.2 \%)$ & \\
\hline pT3 - pT4 & $85(48.6 \%)$ & $32(48.5 \%)$ & \\
\hline \multicolumn{4}{|c|}{$\begin{array}{l}\text { Postoperative Distant and Nodal Disease } \\
\text { (Based on Cystectomy Specimen) (N, \%) }\end{array}$} \\
\hline$\leq$ pNOMO & $125(71.4 \%)$ & $47(71.2 \%)$ & \multirow[t]{2}{*}{1.00} \\
\hline$>$ pNOM0 & $50(28.6 \%)$ & $19(28.8 \%)$ & \\
\hline Diversion Type $(N, \%)$ & & & \\
\hline Ileal Conduit & $145(82.9 \%)$ & $50(75.8 \%)$ & 0.34 \\
\hline Neobladder & $29(16.6 \%)$ & $15(22.7 \%)$ & \\
\hline BMI Category $(N, \%)$ & & & \\
\hline Not Obese ( 30 and below) & $89(50.9 \%)$ & $32(48.5 \%)$ & 0.05 \\
\hline Obese $(30-40)$ & $52(29.7 \%)$ & $16(24.2 \%)$ & \\
\hline Morbidly Obese (40+) & 0 & $2(3 \%)$ & \\
\hline
\end{tabular}

worsening to CKD 3-5 in the immediate postoperative period, though statistical significance was not achieved (OR 2.13, CI 0.93-4.86, $p=0.07$ ). NAC receipt was not associated with worsening to CKD 3-5 stage at 6-12 months follow-up (OR 2.233, $p=0.136$ ).

To better characterize the timeline of renal function loss, we graphically report the GFR of the entire cohort at each time point (Fig. 1). The loss of GFR in the NAC cohort occurs up-front with chemotherapy (mean GFR pre-chemo [73.9], pre-op [65.5], 6-12 month follow-up [59.2]), while patients who underwent cystectomy alone had stable GFR (mean GFR pre-op [63.4], 6-12 month follow-up [62]).

Lastly, we looked at the small subset of 16 patients who had abnormal renal function prior to administration of neoadjuvant chemotherapy. Of those 16 patients, 15 patients were CKD stage 3 , one patient 
was CKD stage 4 , and none were CKD stage 5. Seven (44\%) received cisplatin based chemotherapy and 8 $(50 \%)$ received carboplatin based chemotherapy. The

Table 2

Renal function (serum creatinine, GFR, CKD stage) over time

\begin{tabular}{lcc}
\hline Time Points & $\begin{array}{c}\text { Cystectomy } \\
\text { (RC) Alone }\end{array}$ & $\begin{array}{c}\text { Neoadjuvant } \\
\text { Chemotherapy + RC }\end{array}$ \\
\hline Pre-Chemotherapy & N/A & $\mathrm{N}=56$ \\
Mean Creatinine (SD) & N/A & $1.1 \pm 0.4$ \\
CKD Stage 1 (n, \%) & N/A & $9(16.1 \%)$ \\
CKD Stage 2 (n, \%) & N/A & $31(55.4 \%)$ \\
CKD Stage 3 (n, \%) & N/A & $15(26.8 \%)$ \\
CKD Stage 4 (n, \%) & N/A & $1(1.8 \%)$ \\
CKD Stage 5 (n, \%) & N/A & 0 \\
Post-Chemo, Pre-Operative & N=172 & $\mathrm{N}=64$ \\
Mean Creatinine (SD) & $1.2 \pm 0.5$ & $1.1 \pm 0.3$ \\
CKD Stage 1 (n, \%) & $13(7.6 \%)$ & $6(9.4 \%)$ \\
CKD Stage 2 (n, \%) & $79(46.2 \%)$ & $33(51.6 \%)$ \\
CKD Stage 3 (n, \%) & $71(41.5 \%)$ & $25(39.1 \%)$ \\
CKD Stage 4 (n, \%) & $6(3.5 \%)$ & 0 \\
CKD Stage 5 (n, \%) & $2(1.2 \%)$ & 0 \\
Post-operative & N=173 & $\mathrm{N}=63$ \\
Mean Creatinine (SD) & $1.3 \pm 0.5$ & $1.2 \pm 0.3$ \\
CKD Stage 1 (n, \%) & $13(7.6 \%)$ & $6(9.5 \%)$ \\
CKD Stage 2 (n, \%) & $72(41.9 \%)$ & $26(41.3 \%)$ \\
CKD Stage 3 (n, \%) & $80(46.5 \%)$ & $30(47.6 \%)$ \\
CKD Stage 4 (n, \%) & $5(2.9 \%)$ & $1(1.6 \%)$ \\
CKD Stage 5 (n, \%) & $2(1.2 \%)$ & 0 \\
6-12 Months Follow-up & N=89 & $\mathrm{N}=32$ \\
Mean Creatinine (SD) & $1.3 \pm 0.6$ & $1.3 \pm 0.4$ \\
CKD Stage 1 (n, \%) & $5(5.6 \%)$ & $2(6.2 \%)$ \\
CKD Stage 2 (n, \%) & $44(49.4 \%)$ & $11(34.4 \%)$ \\
CKD Stage 3 (n, \%) & $35(39.3 \%)$ & $19(59.4 \%)$ \\
CKD Stage 4 (n, \%) & $3(3.4 \%)$ & 0 \\
CKD Stage 5 (n, \%) & $2(2.2 \%)$ & 0 \\
\hline
\end{tabular}

patient who was CKD stage 4 pre-chemotherapy had improvement to CKD stage 2 post-operatively. Of the 15 NAC patients who were Stage 3 CKD prior to chemotherapy, one patient progressed to CKD stage 4 post-operatively. Of the 6 patients whose data was available at 6-12 months, all were stable at CKD 3.

\section{DISCUSSION}

Utilization of neoadjuvant chemotherapy remains low despite Level 1 evidence demonstrating a survival benefit for patients with localized, muscle-invasive urothelial carcinoma of the bladder. While underutilization is likely multi-factorial, the concern for chemotherapy induced nephrotoxic injury prior to radical cystectomy may contribute to this trend.

To our knowledge, there are no other studies assessing the effect of neoadjuvant chemotherapy on renal function in this patient population. Despite the fact that the chemotherapeutic agents utilized in each of these platinum-based regimens have known nephrotoxicity and that cystectomy itself carries a risk of renal deterioration [11-14], there is no evidence to support the concern that both utilized in the same patient population leads to worsened overall renal function.

Our analysis demonstrates that neoadjuvant chemotherapy is an independent predictor of renal function deterioration from pre-treatment levels to the post-operative time point, and in patients who received cisplatin based therapy, to the 6-12 month

Table 3A

Multivariable logistic regression analysis of worsening by at least one CKD stage

\begin{tabular}{lcccccc}
\hline & \multicolumn{2}{c}{ Baseline to Post-operative } & & \multicolumn{2}{c}{ Baseline to 6-12 months Follow-up } \\
\cline { 2 - 2 } Covariate & Odds Ratio $(95 \%$ CI $)$ & & $P$-Value & & Odds Ratio (95\% CI) & $P$-Value \\
\hline Neoadjuvant Chemo Received (Yes vs. No) & $2.486(1.248,4.922)$ & & 0.009 & & $2.497(0.992,6.282)$ & 0.050 \\
History of DM: Yes vs. No & $0.849(0.306,2.131)$ & & 0.739 & & $1.426(0.386,4.828)$ & 0.576 \\
Diversion: Neobladder vs. Ileal Conduit & $1.341(0.571,3.031)$ & 0.488 & & $1.916(0.748,4.847)$ & 0.169 \\
History of HTN: Yes vs. No & $1.251(0.622,2.570)$ & & 0.535 & & $1.438(0.593,3.606)$ & 0.427 \\
\hline
\end{tabular}

Table 3B

Multivariable logistic regression analysis of worsening by at least one CKD stage, separated by platinum-based NAC agent

\begin{tabular}{|c|c|c|c|c|}
\hline \multirow[b]{2}{*}{ Covariate } & \multicolumn{2}{|c|}{ Baseline to Post-operative } & \multicolumn{2}{|c|}{ Baseline to 6-12 months Follow-up* } \\
\hline & Odds Ratio (95\% CI) & $P$-Value & Odds Ratio (95\% CI) & $P$-Value \\
\hline Neoadjuvant Chemo: Cisplatin vs. None & $3.726(1.702,8.199)$ & 0.001 & $3.313(1.243,9.046)$ & 0.017 \\
\hline Neoadjuvant Chemo: Carboplatin vs. None & $0.301(0.016,1.651)$ & 0.261 & $0.223(0.002,2.023)$ & 0.219 \\
\hline Neoadjuvant Chemo: Cisplatin vs. Carboplatin & $12.39(2.026,242)$ & 0.023 & $14.85(1.449,2026)$ & 0.019 \\
\hline Age at Surgery (Years) & $0.968(0.930,1.007)$ & 0.109 & $0.979(0.933,1.027)$ & 0.380 \\
\hline History of DM: Yes vs. No & $0.611(0.201,1.621)$ & 0.347 & $1.418(0.396,4.762)$ & 0.579 \\
\hline Diversion: Neobladder vs. Ileal Conduit & $1.405(0.5823 .267)$ & 0.438 & $1.575(0.590,4.119)$ & 0.359 \\
\hline EBL $(100 \mathrm{~mL})$ & $1.011(0.974,1.047)$ & 0.542 & $0.991(0.929,1.038)$ & 0.726 \\
\hline History of HTN: Yes vs. No & $1.331(0.633,2.867)$ & 0.456 & $1.438(0.573,3.752)$ & 0.442 \\
\hline
\end{tabular}

*Analysis for 6-12 months conducted using Firth penalized logistic regression, as there were no events in the carboplatin group that precluded the use of conventional logistic regression. 


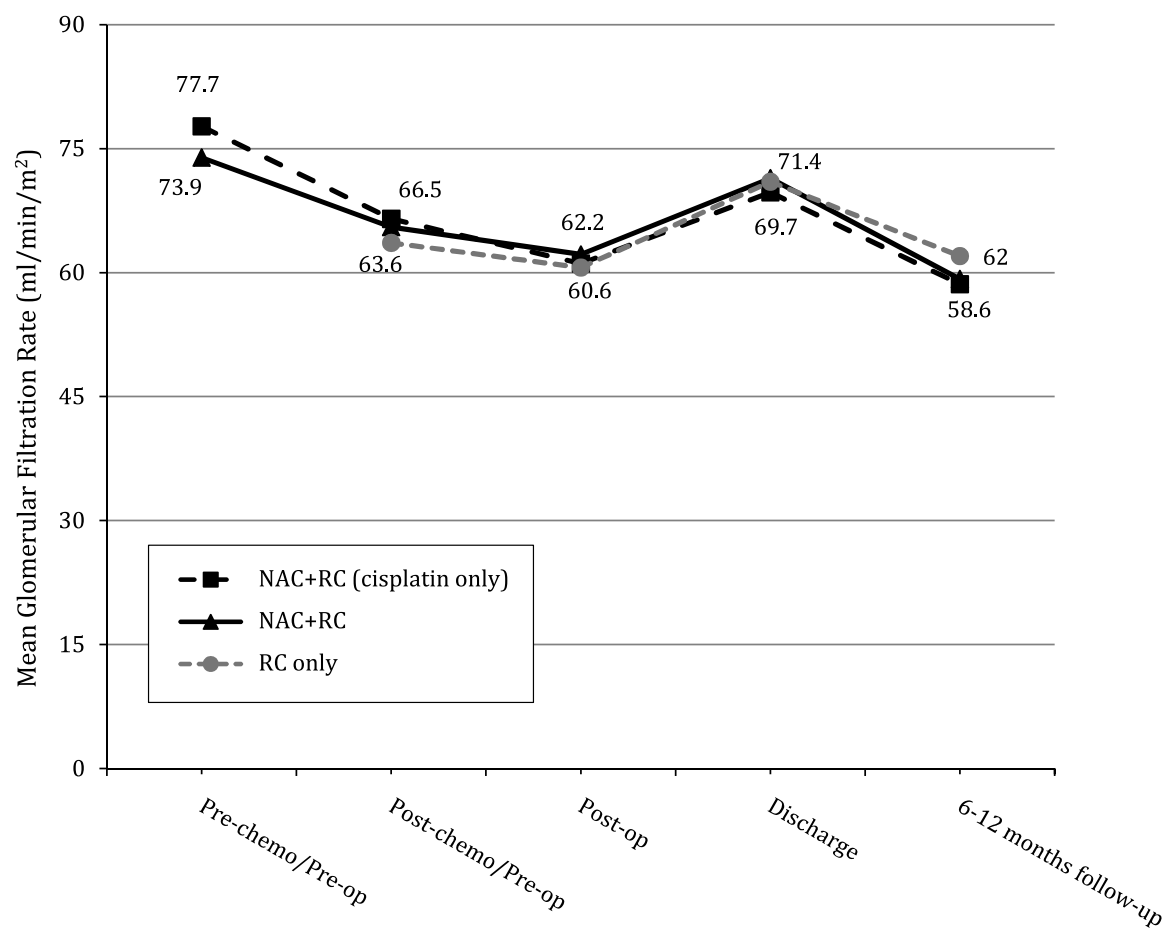

Fig. 1. Mean Glomerular Filtration Rate (GFR) over Time.

follow-up time point. However, single CKD stage deterioration isn't necessarily clinically relevant. As such, we then specifically analyzed deterioration from CKD stage 1-2 to CKD stage 3-5, as a more relevant clinical outcome. In this analysis, NAC was not a predictor of renal function deterioration at the postoperative or 6-12 month follow-up time point. Of note, we were unable to assess cisplatin-based NAC vs. carboplatin-based NAC in this analysis, as the majority of patients in this group received cisplatinbased NAC. The trend towards association to the post-operative time point should be noted, however $(p=0.07)$. This suggests that NAC is not associated with longer-term renal function deterioration, though the patients do take an initial up-front nephrotoxic insult.

This was further assessed by looking at GFR trends. As Fig. 1 demonstrates, there is an initial insult with neoadjuvant chemotherapy administration. However, the peri-operative renal function trends are similar in both cohorts, and the 6-12 month follow-up renal function remains similar. Indeed, even in patients who received cisplatin based NAC only (excluding carboplatin chemotherapy), the same trend in renal function is noted. Of note, the GFR values for our RC alone cohort are consistent with prior studies that examined the effect of cystectomy on long-term renal function; pre-operative GFR ranged from $64.1-77.7 \mathrm{~mL} / \mathrm{min} / 1.73 \mathrm{~m}^{2}$ and last follow-up GFR ranged from $59-69.6 \mathrm{~mL} / \mathrm{min} / 1.73 \mathrm{~m}$ [11-14]. As such, neoadjuvant chemotherapy does not appear to exacerbate the known insult to renal function mediated by cystectomy alone.

Lastly, when we looked at the small subset of 16 patients who had abnormal renal function (CKD stage 3-5) prior to administration of NAC, there was minimal progression of disease. Of the 6 patients whose data was available at 6-12 months, all were stable at CKD 3, and no patients were on dialysis at last follow-up. Nicholson $\mathrm{S}$ notes that all studies on NAC were limited to patients with creatinine clearance $>60 \mathrm{~mL} / \mathrm{min}$, and therefore the guidelines only recommend its administration in patients with adequate renal function. However, there is evidence from the metastatic bladder cancer literature that patients with creatinine clearance $<60 \mathrm{~mL} / \mathrm{min}$ can safely be administered gemcitabine and divided-dose cisplatin $[16,17]$. More recent literature has started to examine utilization of this regimen in the neoadjuvant setting with promising results $[18,19]$. These findings corroborate findings from our study - NAC does not sensitize patients to additional renal function deterioration compared to cystectomy alone, as patients with poor initial renal function were able to tolerate 
platinum-based chemotherapy and still undergo cystectomy without any clinically significant worsening of renal function.

Our data suggests that administration of NAC may cause an initial insult to renal function, but their longterm renal function is no different than patients who undergo RC alone. Indeed, their peri-operative and 6-12 month follow-up renal function follows a similar course to patients undergoing RC alone.

However, our conclusions must be accepted within the context of the limitations of the study. First, as a single-institution retrospective study, we are limited by the sample size of our cohort and retrospective nature of the study. There is selection bias in the cohort, as the decision to administer NAC was physician specific. However, as most of the surgeons at our institution have similar practice patterns, NAC utilization was likely similar. While the referral for NAC may have been consistent, NAC regimen administered was not - this is due to the fact that our patients are often referred to local medical oncologists for NAC administration prior to surgical intervention. As such, the NAC regimen could not be standardized [7, $8,20,21]$. In addition, of the patient's whose regimen was clearly documented, there was insufficient volume to power a comparison between MVAC and GC regimens. Furthermore, with regards to patient selection, inherent to this is the possibility that patients referred for NAC who had higher baseline creatinine and lower GFR may have received carboplatin-based NAC or no chemotherapy at all. This is offset to some degree by the fact that $36.6 \%$ of patients in the RC alone cohort had NMIBC and were not candidates for NAC, and indeed, the pre-operative GFR is similar in both cohorts. Also, as our question primarily looks at the effect of NAC on renal function in relation to RC, we demonstrate that NAC does not add any additional renal function injury to $\mathrm{RC}$ alone in the perioperative setting and at 6-12 month follow-up.

Secondly, as a tertiary referral center, the greatest data capture was in the perioperative period (pre-operative and post-operative values), and our extended follow-up ( $>1$ year) was limited. Our lack of long-term follow-up for both cohorts precludes any ability to determine the long-term consequences of both chemotherapy and cystectomy beyond twelve months. Ultimately, it is the long-term renal function outcomes that will be important to determine if NAC independently affects renal function, taking into account the renal deterioration that can be seen following RC alone. As noted by Samuel JD et al, $29 \%$ of patients following ileal conduit urinary diversion had deterioration of renal function, with no surgical cause identified in $18 \%$ of patients [12]. Age-related decline alone is thought to account for $0.58-1 \mathrm{~mL} / \mathrm{min} / 1.73$ $\mathrm{m}^{2}$ per annum [22-24]. Twenty percent of patients eligible for chemotherapy prior to cystectomy will be ineligible for adjuvant chemotherapy due to renal deterioration [25], which is more reason to ensure all eligible patients undergo neoadjuvant chemotherapy.

While the above data has its limitations, our initial assessment of this patient population suggests that administration of NAC prior to RC does not cause significant renal function deterioration in short term follow-up. There is an initial loss of renal function with NAC administration, but the effect is minimal - downgrading of one CKD stage by the time of 6-12 month follow-up. However, NAC administration is not associated with clinically significant deterioration from CKD stage 1-2 to CKD stage 3-4 at the 6-12 month follow-up time period.

As such, based on this data, there is currently no strong evidence to warrant the concern for renal function deterioration as a reason to not give NAC prior to $\mathrm{RC}$ for eligible patients. Indeed, NAC receipt did not sensitize patients to any greater renal function insult that undergoing cystectomy alone. Level 1 evidence recommends giving NAC to all eligible patients, and our data supports its administration in all eligible patients independent of concern for nephrotoxicity. Further long-term data is required to affirm these findings.

\section{CONFLICTS OF INTEREST}

None.

\section{ACKNOWLEDGMENTS}

The project described was supported by the National Center for Advancing Translational Sciences, National Institutes of Health, through grant number UL1 TR000002. The content is solely the responsibility of the authors and does not necessarily represent the official views of the NIH.

\section{DISCLOSURE STATEMENT}

Dr. Yap reports grants from National Center for Advancing Translational Sciences, National Institutes of Health, during the conduct of the study. 

Dr. Chandrasekar has nothing to disclose.
Dr. Pugashetti has nothing to disclose.
Dr. Durbin-Johnson has nothing to disclose.
Dr. Dall'Era has nothing to disclose.
Dr. Evans has nothing to disclose.
Dr. DeVere White has nothing to disclose.

\section{SUPPLEMENTARY MATERIAL}

The supplementary material is available in the electronic version of this article: http://dx.doi.org/ 10.3233/BLC-160071.

\section{REFERENCES}

[1] Siegel RL, Miller KD, Jemal A. Cancer statistics, 2016. CA Cancer J Clin 2016;66(1):7-30.

[2] Meeks JJ, et al. A systematic review of neoadjuvant and adjuvant chemotherapy for muscle-invasive bladder cancer. Eur Urol 2012; 62(3):523-33.

[3] Dash A, et al. A role for neoadjuvant gemcitabine plus cisplatin in muscle-invasive urothelial carcinoma of the bladder: A retrospective experience. Cancer 2008;113(9): 2471-7.

[4] van de Putte EE, et al. Neoadjuvant induction dosedense MVAC for muscle invasive bladder cancer: Efficacy and safety compared with classic MVAC and gemcitabine/cisplatin. World J Urol 2016;34(2):157-62.

[5] Sio TT, et al. Chemotherapeutic and targeted biological agents for metastatic bladder cancer: A comprehensive review. Int J Urol 2014;21(7):630-7.

[6] Nicholson S. Chemotherapy for bladder cancer in patients with impaired renal function. Nat Rev Urol 2012;9(1):52-7.

[7] Zaid HB, et al. Trends in the utilization of neoadjuvant chemotherapy in muscle-invasive bladder cancer: Results from the National Cancer Database. Urology 2014;83(1): $75-80$.

[8] Krabbe LM, et al. Changing trends in utilization of neoadjuvant chemotherapy in muscle-invasive bladder cancer. Can J Urol 2015;22(4):7865-75.

[9] Cowan NG, et al. Neoadjuvant chemotherapy use in bladder cancer: A survey of current practice and opinions. Adv Urol 2014;2014:746298.

[10] deVere White RW, Katz MH, Steinberg GD. The case for neoadjuvant chemotherapy and cystectomy for muscle invasive bladder cancer. J Urol 2009;181(5):1994-7.
[11] Osawa T, et al. Long-term renal function outcomes in bladder cancer after radical cystectomy. Urol J 2013;10(1): 784-9.

[12] Samuel JD, et al. The natural history of postoperative renal function in patients undergoing ileal conduit diversion for cancer measured using serial isotopic glomerular filtration rate and $99 \mathrm{~m}$ technetium-mercaptoacetyltriglycine renography. J Urol 2006;176(6 Pt 1):2518-22; discussion 2522.

[13] Gershman B, et al. Comparative impact of continent and incontinent urinary diversion on long-term renal function after radical cystectomy in patients with preoperative chronic kidney disease 2 and chronic kidney disease $3 \mathrm{a}$. Int J Urol 2015;22(7):651-6.

[14] Rouanne M, et al. Trends in renal function after radical cystectomy and ileal conduit diversion: New insights regarding estimated glomerular filtration rate variations. Clin Genitourin Cancer 2015;13(3):e139-44.

[15] K/DOQI clinical practice guidelines for chronic kidney disease: Evaluation, classification, and stratification. Am J Kidney Dis 2002;39(2 Suppl 1):S1-266.

[16] Carles J, et al. Feasiblity study of gemcitabine and cisplatin administered every two weeks in patients with advanced urothelial tumors and impaired renal function. Clin Transl Oncol 2006;8(10):755-7.

[17] Hussain SA, et al. A phase I/II study of gemcitabine and fractionated cisplatin in an outpatient setting using a 21-day schedule in patients with advanced and metastatic bladder cancer. Br J Cancer 2004;91(5):844-9.

[18] Hussain SA, et al. A study of split-dose cisplatin-based neoadjuvant chemotherapy in muscle-invasive bladder cancer. Oncol Lett 2012;3(4):855-859.

[19] Vashistha V, et al. Current and recent clinical trials for perioperative systemic therapy for muscle invasive bladder cancer: A systematic review. BMC Cancer 2014;14:966.

[20] Reardon ZD, et al. Trends in the use of perioperative chemotherapy for localized and locally advanced muscleinvasive bladder cancer: A sign of changing tides. Eur Urol 2015;67(1):165-70.

[21] Apolo AB, et al. Examining the management of muscleinvasive bladder cancer by medical oncologists in the United States. Urol Oncol 2014;32(5):637-44.

[22] Weinstein JR, Anderson S. The aging kidney: Physiological changes. Adv Chronic Kidney Dis 2010;17(4):302-7.

[23] Davies DF, Shock NW. Age changes in glomerular filtration rate, effective renal plasma flow, and tubular excretory capacity in adult males. J Clin Invest 1950;29(5):496-507.

[24] Rowe JW, et al. The effect of age on creatinine clearance in men: A cross-sectional and longitudinal study. J Gerontol 1976;31(2):155-63.

[25] Thompson RH, et al. Eligibility for neoadjuvant/adjuvant cisplatin-based chemotherapy among radical cystectomy patients. BJU Int 2014;113(5b):E17-21. 